\title{
Multicenter comprehensive methodological and technical analysis of 832 pressurized intraperitoneal aerosol chemotherapy (PIPAC) interventions performed in 349 patients for peritoneal carcinomatosis treatment: An international survey study
}

\author{
Maciej Nowacki ${ }^{\text {a, * }}$, Mohammad Alyami ${ }^{\text {b, d, e, m, Laurent Villeneuve }}{ }^{\text {c, d, e }}$, \\ Frederic Mercier ${ }^{\mathrm{b}, \mathrm{e}}{ }^{\text {, }}$, Martin Hubner ${ }^{\mathrm{f}}$, Wouter Willaert ${ }^{\mathrm{g}}$, Wim Ceelen ${ }^{\mathrm{g}}$, Marc Reymond ${ }^{\mathrm{h}}$, \\ Denis Pezet ${ }^{\mathrm{i}}$, Catherine Arvieux ${ }^{\mathrm{j}}$, Vladimir Khomyakov ${ }^{\mathrm{k}}$, Laura Lay ${ }^{1}$, Sergio Gianni ${ }^{\mathrm{n}}$, \\ Wojciech Zegarski ${ }^{\text {a }}$, Naoual Bakrin ${ }^{\text {b, d, e }}$, Olivier Glehen ${ }^{\text {b, d, e }}$ \\ ${ }^{\text {a } C h a i r}$ and Department of Surgical Oncology, Ludwik Rydygier's Collegium Medicum in Bydgoszcz, Nicolaus Copernicus University in Torun, Oncology \\ Centre-Prof. Franciszek Lukaszczyk Memorial Hospital in Bydgoszcz, Bydgoszcz, Poland \\ ${ }^{\mathrm{b}}$ Department of Digestive Surgery, Centre Hospitalier Lyon Sud, Pierre-Bénite, France \\ ${ }^{c}$ Hospices Civils de Lyon, Pôle Information Médicale Evaluation Recherche, Unité de Recherche Clinique, Lyon, France \\ ${ }^{\mathrm{d}}$ EMR 3738, Lyon 1 University, Lyon, France \\ e RENAPE, Hospices Civils de Lyon, Centre Hospitalier Lyon Sud, 165 Chemin du Grand Revoyet, 69495, Pierre-Bénite, France \\ ${ }^{\mathrm{f}}$ Department of Visceral Surgery, University Hospital of Lausanne (CHUV), Lausanne, Switzerland \\ ${ }^{g}$ Department of Gastrointestinal Surgery, Ghent University Hospital, Ghent, Belgium \\ h Department of Surgery, University of Tübingen, Tübingen, Germany \\ ${ }^{\mathrm{i}}$ Chirurgie et Oncologie Digestive, Université Clermont Auvergne Clermont-Ferrand, France \\ j Digestive and Emergency Surgery, UGA-Université Grenoble Alpes, Grenoble, France \\ ${ }^{\mathrm{k}}$ Moscow Research Oncological Institute n.a. P.A. Herzen, Thoracoabdominal, Moscow, Russian Federation \\ ${ }^{1}$ Department of Gynecology Surgical Area at the Institute of Oncology A. H. Roffo, University of Buenos Aires, Buenos Aires, Argentina \\ ${ }^{\mathrm{m}}$ King Salman Scholarship Program, Saudi Arabian Cultural Bureau, Paris, France \\ ${ }^{\mathrm{n}}$ Instituto de Oncología Ángel Roffo, Buenos Aires, Argentina
}

\section{A R T I C L E I N F O}

\section{Article history:}

Accepted 15 February 2018

Available online 22 February 2018

\section{Keywords:}

Peritoneal carcinomatosis

PIPAC

Practice survey

Safety procedure

Innovation

Multicenter study

\begin{abstract}
A B S T R A C T
Background: Pressurized intraperitoneal aerosol chemotherapy (PIPAC) is a new drug delivery method offered in selected patients suffering from non-resectable peritoneal carcinomatosis (PC). As reported experience is still limited, we conducted a survey among active PIPAC centers aiming to report their technical approach and clinical findings.

Methods: An online survey was sent to active PIPAC centers worldwide. The questionnaire consisted of 34 closed questions and was conducted over a period of 3 months beginning in March 2017.

Results: Nine out of 15 contacted centers completed the questionnaire totaling 832 PIPAC procedures in 349 patients. Most common indications for PIPAC were PC from gastric, ovarian and colorectal origin. The mean time between each PIPAC procedure was 6-8 weeks. Seven of nine $(77.8 \%)$ centers evaluate the PCI at every PIPAC procedure. At least four tissue samples for histopathology analysis were retrieved in 5 (55.6\%). All centers (100\%) use the same chemotherapy protocol: oxaliplatin at a dosage of $92 \mathrm{mg} / \mathrm{m}^{2}$ for PC of colorectal origin and a combination of cisplatin and doxorubicin at a dosage of $7.5 \mathrm{mg} / \mathrm{m}^{2}$ and $1.5 \mathrm{mg} / \mathrm{m}^{2}$, respectively, for other types of PC. Eight centers $(88.9 \%)$ perform routine radiological evaluation before first PIPAC and after third PIPAC.

Conclusion: These data confirm that PIPAC procedures are homogeneously performed in established centers. Standardization of the procedure will facilitate future international multicenter prospective clinical trials.

๑) 2018 Elsevier Ltd, BASO The Association for Cancer Surgery, and the European Society of Surgical Oncology. All rights reserved.
\end{abstract}

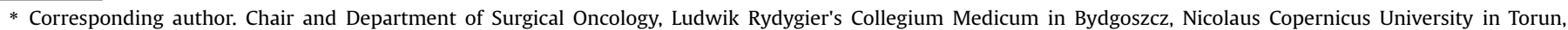
Oncology Centre-Prof. Franciszek Łukaszczyk Memorial Hospital in Bydgoszcz. ul. Romanowskiej 2, 85-796 Bydgoszcz, Romanowskiej 2 str., 85-067 Bydgoszcz, Poland.

E-mail address: maciej.s.nowacki@gmail.com (M. Nowacki).
} 


\section{Introduction}

Pressurized intraperitoneal aerosol chemotherapy (PIPAC) has been used in human for the first time in 2011 [1]. This novel treatment modality allows intraabdominal drug delivery by laparoscopy, entailing pharmacokinetic advantages of better distribution and tissue penetration; Resulting in higher target tissue concentrations of cytostatics as compared with systemic administration or intraabdominal lavage [2,3]. This treatment method of peritoneal carcinomatosis (PC) has been previously tested in several in vitro and in vivo studies [4,5]. Feasibility, safety and tolerance have been described in several studies already and preliminary data on oncological efficacy are encouraging at least [6]. The pioneer group from Reymond et al. provided clear recommendations with regards to operative technique, safety checklist and treatment protocols [7,8]. Due to promising results, PIPAC technology was rapidly adopted and it would be desirable to maintain homogeneous conditions for patient safety but also for clinical research purpose.

The present study aimed therefore to evaluate the different aspects of PIPAC treatment among expert centers worldwide using an online survey.

\section{Methods}

\section{Data collect}

The present survey (online appendix) was elaborated by the authors (MN, MA, OG) and sent electronically to actively involved PIPAC centers globally. The survey was on a voluntary basis. A secure digital platform was used (Surveymonkey Inc. San Mateo, USA) to avoid transcription errors. The survey was reviewed by 2 surgeons (MN, MA), it consisted of 34 closed questions separated in 2 sections. The first section evaluated demographic information and patient population (5 questions). The second part evaluated surgical technique, safety protocol, perioperative strategy and treatment protocols (online appendix). The survey was conducted over a period of 3 months, starting in March 2017. The study was performed in accordance with the precepts established by the declaration of helsinki.

\section{Statistical analysis}

A descriptive statistical analysis was carried out and described quantitative and qualitative data according to means (+/ - standard deviation), medians (range) and percentages. The percentages were calculated over all the responses received for each question.

\section{Results}

\section{Respondents}

Fifteen PIPAC centers were contacted. Three of them did not respond to the request and three responding centers answered only the first question. Final analysis included 9 centers (60\%) from six countries: Argentina, Belgium, France, Germany, Russia and Switzerland.

\section{Etiology}

The data totaled 832 PIPAC procedures performed in 349 patients. Indications for PIPAC are displayed in Table 1.
Table 1

Responses for questions regarding volume and different type of peritoneal carcinomatosis treated. PIPAC - pressurized intraperitoneal aerosol chemotherapy.

\begin{tabular}{lll}
\hline Questions & Answer & Response rate \\
\hline 1- How many patients have been treated with & 349 patients & $100 \%$ \\
PIPAC by your team until now? & & \\
2- How many PIPAC procedures has your team & 832 PIPAC & $100 \%$ \\
performed until now? & & $100 \%$ \\
3- Which types of peritoneal malignancies have & & \\
you treated by PIPAC? & & \\
Gastric & 9 centers & \\
Colorectal & 7 centers & \\
Ovarian & 7 centers & \\
Appendix & 6 centers & \\
Peritoneal mesothelioma & 7 centers & \\
Other & 6 centers & \\
4- How many PIPAC have you done in each type & 680 PIPAC & $80.7 \%$ \\
of peritoneal carcinomatosis? & & \\
Gastric & 280 PIPAC & $41.1 \%$ \\
Colorectal & 137 PIPAC & $20.1 \%$ \\
Ovarian & 154 PIPAC & $22.7 \%$ \\
Appendix & 38 PIPAC & $5.6 \%$ \\
Peritoneal mesothelioma & 34 PIPAC & $5 \%$ \\
Other & 37 PIPAC & $5.5 \%$ \\
\hline
\end{tabular}

\section{PIPAC conditions (Table 2)}

PIPAC procedure was mainly performed using supine position (89\%). Only one center performed PIPAC with spread leg holders (11\%). In most of the centers, the surgeon operated on the right side with the assistant on the left side of the patient (55,6\%). Five centers (55.6\%) used mini-laparotomy (open technique) to insert the first trocar. Remaining centers (44.4\%) used the Veress needle technique. All centers used two trocars of 12,10 or $5 \mathrm{~mm}$ size and there was no difference noted according to the first and repetitive PIPAC trocar insertion technique. Double balloon trocars were used in 6 centers (66,7\%), single balloon trocars in 2 centers $(22.1 \%)$ and trocars without balloon system in one center $(11,2 \%)$.

\section{Trocars placement}

The majority of centers (55.6\%) placed them in the midline, two (22.2\%) centers laterally and two (22.2\%) center placed them depending on prior surgical history (either midline or laterally).

\section{Protective equipment}

Five (55.6\%) centers used, during port placement, an additional protection for unexpected leak either sutures or plastic cover connected to smoke filtration device. The operating room was equipped with laminar airflow in 6 (66,7\%) centers. In 7 (77.8\%) centers, there was no difference in ports placement related to the origin of the PC (Table 2).

\section{Timeline of PIPAC procedures}

Mean time interval between each PIPAC procedure was 6 weeks and 8 weeks, in $7(77.8 \%)$ and $2(22.2 \%)$ centers, respectively.

The highest number of PIPAC procedures performed in the same patient was 9 .

\section{Peritoneal cytology}

In five (55.6\%) centers, a cytology sampling from ascites was performed at each PIPAC. Those five centers have reported cytology sampling procedure, even when there is no ascites, using a flush cytology technique. 
Table 2

Responses on questions regarding PIPAC technique. PIPAC-pressurized intraperitoneal aerosol chemotherapy.

\begin{tabular}{|c|c|c|}
\hline Questions & Answer & Response rate \\
\hline 1- What types of patient's positioning are you using? & & $100 \%$ \\
\hline Classic supine & 8 centers $(89.9 \%)$ & \\
\hline With spread leg holders & 1 center $(11.1 \%)$ & \\
\hline 2-What is the position the surgical team? & & $100 \%$ \\
\hline - Surgeon & & \\
\hline$\circ$ Left side & 5 & \\
\hline ○ Right side & 3 & \\
\hline - Between leg & 1 & \\
\hline - Assistant & & \\
\hline$\circ$ Left side & 5 & \\
\hline$\circ$ Right side & 4 & \\
\hline 3-Which method do you use to perform pneumoperitoneum and to properly place first trocar? & & $100 \%$ \\
\hline Mini-laparotomy & 5 & \\
\hline Veress needle & 4 & \\
\hline 4- How many trocar/ports do you use? & 2 trocars & $100 \%$ \\
\hline 5- What sizes of trocar/ports do you use? & & $100 \%$ \\
\hline Size 5 & 1 center & \\
\hline Size 10 & 5 centers & \\
\hline Size 11 & 1 center & \\
\hline Size 12 & 2 centers & \\
\hline 6- What type(s) of trocar(s) do you use? & & $100 \%$ \\
\hline Double balloon & 6 centers & \\
\hline Single balloon & 2 centers & \\
\hline Usual trocars & 1 center & \\
\hline 7-Where do you position trocar's/ports? & & $100 \%$ \\
\hline Midline & 5 centers & \\
\hline Lateral & 2 centers & \\
\hline Both & 2 centers & \\
\hline 8-Do you use other additional protection for unexpected leak? i.e. additional sutures or tools & & $100 \%$ \\
\hline - No & 4 & \\
\hline - Yes & 5 & \\
\hline$\circ$ Sutures if necessary & 3 & \\
\hline$\circ$ Plastic cover + smoke evacuation device + sutures if necessary & 2 & \\
\hline 9-Does your operating room is equipped with laminar airflow? & & $100 \%$ \\
\hline No & 3 & \\
\hline Yes & 6 & \\
\hline 10-How do you do the evacuation of the remaining toxic aerosol at the end of PIPAC? & & $100 \%$ \\
\hline - Through microparticle filters into the air filtration system of the hospital & 5 & \\
\hline - Through closed surgical smoke evacuation system & 4 & \\
\hline 11-Is there any difference in laparoscopy preparations or ports placement according to the peritoneal carcinomatosis etiology? & & $100 \%$ \\
\hline - No & 7 & \\
\hline - Yes & 2 & \\
\hline $\begin{array}{l}\text { 12-Are there any difference in laparoscopy preparations or ports placement according to the number of procedures performed } \\
\text { on the same patient? i.e. do you change your PIPAC preparation } \\
\text { protocol if this is a second, third, etc. PIPAC procedure performed in the same patient. }\end{array}$ & & $100 \%$ \\
\hline - No & 8 & \\
\hline - Yes & 1 & \\
\hline
\end{tabular}

Assessment of the peritoneal carcinomatosis extent

Seven (77.8\%) center always evaluated the peritoneal cancer index $(\mathrm{PCI})$ during each PIPAC procedure. Four or five biopsies were done in 5 (55.6\%) centers and in the remaining centers they collected one to three biopsies. The biopsies were performed on the largest/principal lesion in four centers (44.4\%), from lesions located in specific anatomic areas in four centers $(44.4 \%)$ and in non-specific areas in one center (11.2\%). In five centers $(55,6 \%)$, normal tissue biopsies were performed systematically.

\section{PIPAC procedure}

Seven centers $(77.8 \%)$ used a double syringe injector system and two centers $(22.2 \%)$ used a single syringe system. Two centers $(22.2 \%)$ did not use any external monitors or display system, they directly observed the procedure via the operating room door.

\section{Chemotherapy regimen}

Regarding chemotherapy agents, all centers (100\%) used the same protocol: oxaliplatin at $92 \mathrm{mg} / \mathrm{m}^{2}$ for PC of colorectal origin and a combination of cisplatin and doxorubicin at a dosage of $7.5 \mathrm{mg} / \mathrm{m}^{2}$ and $1.5 \mathrm{mg} / \mathrm{m}^{2}$, respectively, for other types of PC. One center (11.2\%) also used a Mitomycin C protocol, at $1.5 \mathrm{mg} / \mathrm{m}^{2}$ dosage in case of formal contraindication to previously mentioned protocols. Seven centers combined PIPAC with various IV chemotherapy protocol. Four centers (44.4\%) measured tumor markers at each PIPAC cycle and 8 centers (88.9\%) performed radiological evaluation before the first PIPAC and after three PIPAC (Table 3).

\section{Discussion}

The present survey indicates that current application of PIPAC as new treatment approach is well standardized with regards to indications, technical aspects, safety protocol and treatment regimens. This is probably explained by a standardized implementation process and the limited number of expert centers. 
Table 3

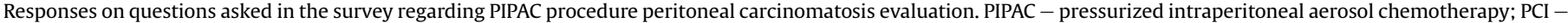
peritoneal carcinomatosis index; PC - peritoneal carcinomatosis; CEA - carcino-embryonic antigen.

\begin{tabular}{|c|c|c|}
\hline Questions & Answer & Response rate \\
\hline 1- What is the mean time (in weeks) between each sequentially performed PIPAC in your institution? & & $100 \%$ \\
\hline 4 weeks & 0 & \\
\hline 6 weeks & 7 & \\
\hline 8 weeks & 2 & \\
\hline $\begin{array}{l}\text { 2- What is the maximal number of PIPAC procedures that you have performed for the same patient? } \\
\text { (If it is possible please describe shortly the case) }\end{array}$ & & $100 \%$ \\
\hline \multicolumn{3}{|l|}{2 PIPAC procedures } \\
\hline 3 PIPAC procedures & 1 center & \\
\hline 4 PIPAC procedures & 1 center & \\
\hline 5 PIPAC procedures & 1 center & \\
\hline 6 PIPAC procedures & 1 center & \\
\hline 7 PIPAC procedures & 3 centers & \\
\hline 8 PIPAC procedures & 1 center & \\
\hline 9 PIPAC procedures & 1 center & \\
\hline 3- Do you always perform a cytology? & & $100 \%$ \\
\hline No & 4 & \\
\hline Yes & 5 & \\
\hline 4- If there is no ascites presented do you inject fluid in order to obtain a cytology ? & & $100 \%$ \\
\hline No & 0 & \\
\hline Yes & 5 & \\
\hline \multicolumn{3}{|l|}{ 5- Do you perform PCI evaluation during each PIPAC procedure? } \\
\hline No & 2 & \\
\hline Yes & 7 & $100 \%$ \\
\hline \multicolumn{3}{|l|}{ 6- Do you use other PC scoring system beside PCI-Sugerbaker index? } \\
\hline No & 9 & \\
\hline Yes & 0 & $100 \%$ \\
\hline 7- How many tissue sample(s) do you usually obtain for the histopathology analysis? & & $100 \%$ \\
\hline 1 & 2 & \\
\hline 2 & 1 & \\
\hline 3 & 1 & \\
\hline 4 & 4 & \\
\hline 5 & 1 & \\
\hline 8- Do you do your biopsy from & & $100 \%$ \\
\hline Main/largest lesions & 4 & \\
\hline Lesions and spots located in specific anatomic areas & 4 & \\
\hline From non-specified anatomical region & 1 & \\
\hline 9- Do you always perform a biopsy from non-invaded peritoneum (local peritonectomy)? & & $100 \%$ \\
\hline No & 4 & \\
\hline Yes & 5 & \\
\hline 10- Which type of injector does you uses? & & $100 \%$ \\
\hline Single syringe & 2 & \\
\hline Double syringe & 7 & \\
\hline 11- Do you use the external monitors and displays? & & $100 \%$ \\
\hline Injector & 7 & \\
\hline Anesthesia & 6 & \\
\hline Lap monitors & 5 & \\
\hline 12-What type chemotherapy (with this dosage) do you use for each type of peritoneal carcinomatosis? & & $100 \%$ \\
\hline Oxaliplatin $92 \mathrm{mg} / \mathrm{m}^{2}$ colorectal & 6 & \\
\hline Cisplatin $7.5 \mathrm{mg} / \mathrm{m}^{2}+$ Doxorubicin $1.5 \mathrm{mg} / \mathrm{m}^{2}$ for other all indication & 8 & \\
\hline Mitomycin or Eloxatine & 1 & \\
\hline 13- In your overall treatment of peritoneal carcinomatosis, do you combine PIPAC with IV chemotherapy? & & $100 \%$ \\
\hline No & 2 & \\
\hline Yes & 7 & \\
\hline $\begin{array}{l}\text { 14- If yes, what is your mean delay between IV chemotherapy cycle and PIPAC procedure? And between } \\
\text { PIPAC procedure and IV chemotherapy cycle? }\end{array}$ & & $100 \%$ \\
\hline \multicolumn{3}{|l|}{ Before PIPAC } \\
\hline 1 week & 1 & \\
\hline 2 weeks & 8 & \\
\hline \multicolumn{3}{|l|}{ After PIPAC } \\
\hline 1 week & 3 & \\
\hline 2 weeks & 6 & \\
\hline 15- Do you evaluate the tumor marker during PIPAC? & & $100 \%$ \\
\hline No & 5 & \\
\hline Yes & 4 & \\
\hline - CEA & 3 & \\
\hline - Ca19.9 & 3 & \\
\hline - Ca125 & 4 & \\
\hline 16-Do you do radiological evaluation before or after PIPAC? & & $100 \%$ \\
\hline No & 1 & \\
\hline Yes & 8 & \\
\hline
\end{tabular}


Up until now, only one institution described, retrospectively, the practical and technical aspects of PIPAC procedure in a large group of patients [8]. Recently, a large number of studies including cohort groups and case report showed promising results, when performed in a selected group of patients with PC $[9,10]$. There is a lack of knowledge regarding the technical implementation of this drug delivery method. Many studies in surgical oncology have shown that proper assessment of treatment needs proper data collection. The goal is to harmonize the treatment between centers, which is important especially when many active pharmacological substances are used [11,12].

The collected data showed that most of the PIPAC procedures were performed in gastric cancer (41.1\%), ovarian cancer $(22.7 \%)$ and colorectal cancer (20.1\%). This probably results from the responding PIPAC centers, mainly specialized in digestive tract surgical oncology. Frequent inability to perform CRS + HIPEC in these 3 primaries leading to PC is another hypothesis $[13,14]$. This study shows that all center performing PIPAC use two trocars for a procedure (one for camera and another for the nebulizer) which is standard in PIPAC without needing the presence of a member of the surgical team in the operating room [15]. The difference between surgical access with the Veress needle method and minilaparotomy could be explained by surgical habits associated with other types of laparoscopic operations performed in each center. There is currently no gold standard in the literature for surgical access. Since no data show the superiority of one of these methods, this topic is still controversial for many surgeons [16]. Some author's report that the Veress needle technique, since it's a blind method, is correlated with a significantly higher risk of intraoperative complications including soft tissue injuries and bleeding [17]. Since any kind of intraperitoneal injury is a formal contraindication to realize the procedure, mini-laparotomy with direct visualization of trocar insertion could be more interesting in PIPAC $[18,19]$. Some centers using mini-laparotomy reported additional procedure to avoid the risk of unexpected $\mathrm{CO}_{2}$ or aerosol leak. Additional sutures are positioned despite the use of double balloon trocars.

Our study showed that there is a significant difference in ports placement between centers, but all center use the same position. Equipment availability of each operating room and philosophy of "minimally invasive" surgery are 2 reasons which could explain this difference. Availability of laparoscopic devices produced and distributed by a wide range of suppliers may influence the surgical team operative technique. Different training or philosophy of "minimal invasive surgery" between surgeons from different institution could also impact operative technique [20,21]. For example, some surgical oncologists always perform the simplest access while others modify the procedure based on patient oncological status or previous surgeries [22].

In every center, the minimal time between treatments was 6 weeks with a maximal delay of 8 weeks. Such time period is reported as optimal in several clinical studies of minimally invasive procedures. In urology, when total prostatectomy is planned after prostate biopsy, there is a waiting period to ensure the safety of surgery. This period lowers the risk of prostate inflammation or contributes to complication healing, which could influence on patient perioperative condition [4,23]. A patient underwent nine PIPAC procedure without any significant complications following this schedule.

Results show that most centers use Reymond et al. management including check list, PCI scoring, safety procedures, laminar air flow, injector system and chemotherapy protocol [7]. In all center, assessment includes several cytology, several biopsies and local peritonectomies to evaluate the effect of therapy. There are only subtle differences in protocol. The number of taken biopsies was at least 4 biopsies for 5 centers in our survey two from the upper abdomen and two from the lower. This issue has to be evaluated in the future to identify the best number and place for the biopsy and the re-biopsy depend on the extent of carcinomatosis and to get a homogenous recommended anatomical region sites, what could be crucial in aspects of re-biopsies and also in case of standardization of pathological response after PIPAC like in other intraperitoneal chemotherapy procedures [24]. Limitations are related to medical equipment purchase differing through hospital (external screen devices or injectors). Homogeneity of PIPAC protocol are fundamental to obtain good data and high level of evidence. Equipment heterogeneity could influence implementation of multicenter trial evaluating PIPAC efficacy $[25,26]$.

The most important issue is the assessment of PIPAC chemotherapy protocols. Every surveyed center follows recommended protocol. Oxaliplatin at $92 \mathrm{mg} / \mathrm{m}^{2}$ for colorectal PC and cisplatin at $7.5 \mathrm{mg} / \mathrm{m}^{2}+$ doxorubicin at $1.5 \mathrm{mg} / \mathrm{m}^{2}$ for other types of PC. These protocols have been established in preclinical studies and evaluated in several clinical studies $[27,28]$. One center $(11,2 \%)$ also use a protocol based on Mitomycin C $\left(1.5 \mathrm{mg} / \mathrm{m}^{2}\right)$ for patients with colorectal PC that cannot receive oxaliplatin for intolerance [29]. For the treatment schedule alyami et al. proposed alternating systemic chemotherapy and PIPAC cycles [30], which seemed feasible and allowed the maintenance of systemic therapy, and Grass et al. confirmed that concomitant PIPAC and systemic therapy appeared to be well tolerated [6]. Only half of surveyed centers used tumor markers assessment but every centers performed detailed radiological evaluation. It was expected since tumor marker levels are not commonly used during PC treatment even if their value has been proven in literature [31].

This study has several limitations. First, only a limited number of PIPAC centers were available at the time of the survey and only $60 \%$ of contacted experts responded. True practice might differ from what was described here. Nonetheless, the expert panel included most major centers, responsible for most of the published data. Second, the current study reports on intended protocols that might differ from actual practice for various reasons as outlined recently [8].

In summary, PIPAC is a standardized procedure that is performed in a small number of expert centers. Mandatory certification courses and structured implementation programs are necessary to maintain the high safety standards for this novel treatment. Moreover, standardized protocols will facilitate collection of homogenous data on PIPAC treatment.

\section{Declaration of interest}

None.

\section{Appendix A. Supplementary data}

Supplementary data related to this article can be found at https://doi.org/10.1016/j.ejso.2018.02.014.

\section{References}

[1] Solass W, Giger-Pabst U, Zieren J, Reymond MA. Pressurized intraperitoneal aerosol chemotherapy (PIPAC): occupational health and safety aspects. Ann Surg Oncol 2013;20(11):3504-11.

[2] Lehmann K, Hübner M. Pressurized intra-peritoneal aerosol chemotherapy (PIPAC): Ein nächster Schritt in der chirurgischen Behand- lung der Peritonealkarzinomatose. Swiss Knife 2015;1:9-11.

[3] Solass W, Kerb R, Murdter T, Giger-Pabst U, Strumberg D, Tempfer C, et al. Intraperitoneal chemotherapy of peritoneal carcinomatosis using pressurized aerosol as an alternative to liquid solution: first evidence for efficacy. Ann Surg Oncol 2014;21(2):553-9.

[4] Teixeira Farinha H, Grass F, Kefleyesus A, Achtari C, Romain B, Montemurro M, et al. Impact of pressurized intraperitoneal aerosol chemotherapy on quality of life and symptoms in patients with peritoneal carcinomatosis: a retrospective cohort study. Gastroenterol Res Pract 2017;2017:4596176. 
[5] Tempfer CB. Pressurized intraperitoneal aerosol chemotherapy as an innovative approach to treat peritoneal carcinomatosis. Med Hypotheses 2015;85(4): 480-4.

[6] Grass F, Vuagniaux A, Teixeira-Farinha H, Lehmann K, Demartines N, Hubner M. Systematic review of pressurized intraperitoneal aerosol chemotherapy for the treatment of advanced peritoneal carcinomatosis. Br J Surg 2017;104(6): 669-78.

[7] Reymond MA, Solass W. PIPAC: pressurized intraperitoneal aerosol chemotherapy: cancer under pressure. Berlin (Germany) Boston (Massachusetts): Walter de Gruyter GmbH; 2014

[8] Hubner M, Grass F, Teixeira-Farinha H, Pache B, Mathevet P, Demartines N. Pressurized intraperitoneal aerosol chemotherapy - practical aspects. Eur J Surg Oncol 2017:43(6):1102-9.

[9] Odendahl K, Solass W, Demtroder C, Giger-Pabst U, Zieren J, Tempfer C, et al. Quality of life of patients with end-stage peritoneal metastasis treated with pressurized intraperitoneal aerosol chemotherapy (PIPAC). Eur J Surg Oncol 2015;41(10):1379-85.

[10] Tempfer CB, Solass W, Buerkle B, Reymond MA. Pressurized intraperitoneal aerosol chemotherapy (PIPAC) with cisplatin and doxorubicin in a woman with pseudomyxoma peritonei: a case report. Gynecol Oncol Rep 2014:10:32-5.

[11] Avigan MI, Bjornsson ES, Pasanen M, Cooper C, Andrade RJ, Watkins PB, et al. Liver safety assessment: required data elements and best practices for data collection and standardization in clinical trials. Drug Saf 2014;37(Suppl 1): S19-31.

[12] Habib SL, Al-Obaidi NY, Nowacki M, Pietkun K, Zegarska B, Kloskowski T, et al. Is mTOR inhibitor good enough for treatment all tumors in TSC patients? J Cancer 2016:7(12):1621-31.

[13] Koppe MJ, Boerman OC, Oyen WJ, Bleichrodt RP. Peritoneal carcinomatosis of colorectal origin: incidence and current treatment strategies. Ann Surg 2006;243(2):212-22

[14] Cardi M, Sammartino P, Mingarelli V, Sibio S, Accarpio F, Biacchi D, et al Cytoreduction and HIPEC in the treatment of "unconventional" secondary peritoneal carcinomatosis. World J Surg Oncol 2015;13:305.

[15] Oyais A, Solass W, Zieren J, Reymond MA, Giger-Pabst U. Occupational health aspects of pressurised intraperitoneal aerosol chemotherapy (PIPAC): confirmation of harmlessness. Zentralbl Chir 2016;141(4):421-4.

[16] Taye MK, Fazal SA, Pegu D, Saikia D. Open versus closed laparoscopy: yet an unresolved controversy. J Clin Diagn Res 2016;10(2):QC04-07.

[17] Toro A, Mannino M, Cappello G, Di Stefano A, Di Carlo I. Comparison of two entry methods for laparoscopic port entry: technical point of view. Diagn Ther Endosc 2012;2012:305428.

[18] Sabaila A, Fauconnier A, Huchon C. Pressurized intraperitoneal aerosol chemotherapy (PIPAC): a new way of administration in peritoneal carcinomatosis of ovarian cancer. Gynecol Obstet Fertil 2015;43(1):66-7.
[19] Alkatout I, Mettler L, Maass N, Noe GK, Elessawy M. Abdominal anatomy in the context of port placement and trocars. J Turk Ger Gynecol Assoc 2015;16(4):241-51.

[20] Dal Moro F, Secco S, Valotto C, Artibani W, Zattoni F. Specific learning curve fo port placement and docking of da Vinci((R)) Surgical System: one surgeon's experience in robotic-assisted radical prostatectomy. J Robot Surg 2012;6(4): $323-7$.

[21] Jain N, Sareen S, Kanawa S, Jain V, Gupta S, Mann S. Jain point: a new safe portal for laparoscopic entry in previous surgery cases. J Hum Reprod Sci 2016;9(1):9-17.

[22] Adiyat KT, Murugesan M, Katkoori D, Eldefrawy A, Soloway MS. Total pros tatectomy within 6 weeks of a prostate biopsy: is it safe? Int Braz J Urol 2010;36(2):177-81. discussion 182.

[23] Gopalakrishnan S, Ganeshkumar P. Systematic reviews and meta-analysis: understanding the best evidence in primary healthcare. J Fam Med Prim Care 2013:2(1):9-14.

[24] Yonemura Y, Canbay E, Ishibashi H. Prognostic factors of peritoneal metastases from colorectal cancer following cytoreductive surgery and perioperative chemotherapy. Sci World J 2013;18(4):978394.

[25] Haidich AB. Meta-analysis in medical research. Hippokratia 2010;14(Suppl 1): 29-37.

[26] Demtroder C, Solass W, Zieren J, Strumberg D, Giger-Pabst U, Reymond MA. Pressurized intraperitoneal aerosol chemotherapy with oxaliplatin in colorectal peritoneal metastasis. Colorectal Dis 2016;18(4):364-71.

[27] Hubner M, Teixeira Farinha H, Grass F, Wolfer A, Mathevet P, Hahnloser D et al. Feasibility and safety of pressurized intraperitoneal aerosol chemotherapy for peritoneal carcinomatosis: a retrospective cohort study. Gastroenterol Res Pract 2017;2017:6852749.

[28] Tempfer CB, Winnekendonk G, Solass W, Horvat R, Giger-Pabst U, Zieren J, et al. Pressurized intraperitoneal aerosol chemotherapy in women with recurrent ovarian cancer: a phase 2 study. Gynecol Oncol 2015;137(2): 223-8.

[29] Hompes D, D'Hoore A, Wolthuis A, Fieuws S, Mirck B, Bruin S, et al. The use of oxaliplatin or mitomycin $C$ in HIPEC treatment for peritoneal carcinomatosis from colorectal cancer: a comparative study. J Surg Oncol 2014;109(6): 527-32.

[30] Alyami M, Gagniere J, Sgarbura O, Cabelguenne D, Villeneuve L, Pezet D, et al Multicentric initial experience with the use of the pressurized intraperitoneal aerosol chemotherapy (PIPAC) in the management of unresectable peritonea carcinomatosis. Eur J Surg Oncol 2017 Nov;43(11):2178-83.

[31] Wagner PL, Austin F, Sathaiah M, Magge D, Maduekwe U, Ramalingam L, et al. Significance of serum tumor marker levels in peritoneal carci carcinomatosis of appendiceal origin. Ann Surg Oncol 2013;20(2):506-14. 